\title{
The effect of SSD, Field size, Energy and Detector type for Relative Output Factor measurement in small photon beams as compared with Monte Carlo simulation
}

\author{
Itumeleng SETILO', Oluwaseyi Michael ODERINDE ${ }^{1, a}$, Freek CP DU PLESSIS ${ }^{1}$ \\ ${ }^{I}$ Department of Medical Physics, University of the Free State, PO Box 339, Bloemfontein 9300, Republic of South Africa \\ ${ }^{a}$ E-mail address: oderindeseyi02@gmail.com
}

(received 12 August 2018; revised 6 October, 25 November 2018 and 24 January 2019; accepted 12 February 2019)

\begin{abstract}
Introduction: Small fields photon dosimetry is associated with many problems. Using the right detector for measurement plays a fundamental role. This study investigated the measurement of relative output for small photon fields with different detectors. It was investigated for three-photon beam energies at SSDs of 90, 95, 100 and $110 \mathrm{~cm}$. As a benchmark, the Monte Carlo simulation was done to calculate the relative output of these small photon beams for the dose in water.

Materials and Methods: 6, 10 and 15 MV beams were delivered from a Synergy LINAC equipped with an Agility 160 multileaf collimator (MLC). A CC01 ion chamber, EFD-3G diode, PTW60019 microdiamond, EBT2 radiochromic film, and EDR2 radiographic film were used to measure the relative output of the linac. Measurements were taken in water for the CC01 ion chamber, EFD-3G diode, and the PTW60019. Films were measured in water equivalent RW3 phantom slabs. Measurements were made for $1 \times 1,2 \times 2,3 \times 3,4 \times 4,5 \times 5$ and a reference field of $10 \times 10 \mathrm{~cm}^{2}$. Field sizes were defined at $100 \mathrm{~cm}$ SSD. Relative output factors were also compared with Monte Carlo (MC) simulation of the LINAC and a water phantom model. The influence of voxel size was also investigated for relative output measurement. Results and Discussion: The relative output factor (ROF) increased with energy for all fields large enough to have lateral electronic equilibrium (LEE). This relation broke down as the field sizes decreased due to the onset of lateral electronic disequilibrium (LED). The high-density detector, PTW60019 gave the highest ROF for the different energies, with the less dense CC01 giving the lowest ROFs.

Conclusion: These are results compared to MC simulation, higher density detectors give higher ROF values. Relative to water, the ROF measured with the air-chamber remained virtually unchanged. The ROFs, as measured in this study showed little variation due to increased SSDs. The effect of voxel size for the Monte Carlo calculations in water does not lead to significant ROF variation over the small fields studied.
\end{abstract}

Key words: small-field dosimetry; relative output factor; electronic equilibrium; Monte Carlo.

\section{Introduction}

Small fields or segments used for radiation dose delivery can be found in IMRT, VMAT, SBRT and SRS techniques [1]. These techniques require a high level of confidence in the accuracy of the entire treatment as high doses can be delivered to the target [2]. However, it is a difficult task to determine experimentally small field dosimetric characteristics due to lateral electronic disequilibrium, high gradient penumbra and sharp peak dose profiles, volume averaging effect of detectors and signal-to-noise ratio of detectors [3].

Fields less than $3 \times 3 \mathrm{~cm}^{2}$ size are considered as small [4,5]. In the small fields, there is no lateral charged particle equilibrium. It means that more electrons move out from the central part of the beam than from the outer part to the center
[6]. The detector used must not disturb the existing LED state of the field [7,8]. Also, there must be a careful consideration in choosing a small field detector since every detector will average the detected dose over its volume. Volume averaging effect will undoubtedly yield a different signal for each detector. Nasir et al. compared the use of CC13 and CC01 ionization chamber for small field ROFs $\left(1 \times 1-5 \times 5 \mathrm{~cm}^{2}\right.$ at $100 \mathrm{~cm} \mathrm{SSD} \mathrm{for} 6$ and $15 \mathrm{MV}$ ). The study shows that the measurements performed with the CC13 chamber underestimates the ROF and are insensitive to a relative error of $22.89 \%$ smallest field considered as compared to CC01 [9].

Accordingly, the detector material will decrease/increase interactions according to its physical density [10]. The perfect detector should be water equivalent $\left(\rho=1 \mathrm{~g} / \mathrm{cm}^{3}\right)$ and should have a small sensitive area to measure the sharp penumbrae of 
small fields accurately. An ideal detector does not exist. Each detector has its advantages and disadvantages. The ROF can be calculated accurately with the Monte Carlo method. Also, it can be used to study the effect of a perfect water-equivalent detector. In this study, a radiographic (EDR2) and radiochromic film (EBT3), an ion chamber (CC01), a diode (EFD3G), and a micro-diamond (PTW60019) detectors were used to measure relative output factors (ROF's) for small fields as recommended [11].

The IBA CC01 ionization chamber (IBA Dosimetry AB Sweden) has a small sensitive air volume of $0.01 \mathrm{cc}$ (Table 1). It has a steel inner electrode to increase the signal to noise ratio [11]. The smaller volume results in better penumbra definition and offers excellent stability. It can be used either in the perpendicular or parallel orientation with an insignificant effect on the resolution [12].

The IBA EFD ${ }^{3 \mathrm{G}}$ Electron diode detector (ScanditronixWellhofer Albertville, USA) is the 3rd generation p-type silicon ( $\mathrm{pSi}$ ) semiconductor which has a small sensitive diameter and thickness as shown in Table 1. It has higher sensitivity compared to ion chambers but suffers from directional dependence and long-term irreversible ionizing radiation damage which alters their sensitivity [13]. Its higher density Silicon sensitive volume requires small amounts of radiation energy for ion pair formation which significantly improves the signal to noise ratio. It is 1800 times denser than air. Warm-up time is not necessary before measurement, and the detector is energy and dose-rate independent.

The PTW60019 micro-diamond (PTW GmbH, Germany) is the first commercially available single crystal diamond detector (SCDD). The synthetic micro-diamond detector is high-density detector which overcomes dose rate dependency and offers the same advantages as diodes but without radiation damage over time. This near water equivalent detector has a minimal sensitive volume of $0.0004 \mathrm{cc}$ and is a good candidate for small-field dosimetry. Study has shown that this detector has minimal energy, temperature, and directional dependency; thus changes within these factors will not influence the measured signal [14].

EDR2 film (KODAK NY, USA) is a radiographic film that uses silver bromide crystals of uniform size with an effective silver thickness of $0.2 \mu \mathrm{m}$. The silver layer density is $2.3 \mathrm{~g} / \mathrm{cm}^{2}$. The film suffers from having an active energy dependence due to its high effective atomic number [4]. It is also sensitive to light which makes the handling it cumbersome and development of the film to be carried out in a dark room. The recommended dose to the film is $500 \mathrm{cGy}$. Literature has shown that Kodak films experience a $5 \%$ reduction in optical density (OD) when the dose rate is decreased by a factor of 12 [15]. To avoid this error, the films were exposed at a constant dose rate of $400 \mathrm{cGy} / \mathrm{min}$ for all measurements.

Gafchromic ${ }^{\circledR}$ EBT2 film (ISP Technologies Inc, USA) is less sensitive to room light but sensitive to ionizing radiation and is self-developing and therefore the result will not be influenced by developer temperature, as it is for the radiographic film [15]. The equivalent photon mass energy absorption coefficients and electron mass collision stopping powers are the same for water [16,17]. It is virtual energy independent with a high spatial resolution due to very small active particles. These particles are needle-like $(15-25 \mu \mathrm{m}$ in length) and 1-2 $\mu \mathrm{m}$ in diameter and [18]. They are sandwiched between a polyester over-laminate $(50 \mu \mathrm{m})$ and a polyester substrate $175 \mu \mathrm{m}$ [19]. The measurement side should be chosen and adhered to due to this difference in the thickness of overlays. A waiting period of 24 hours post-irradiation is recommended to allow for proper film development and stabilization due to post-irradiation polymerization.

Monte Carlo (MC) simulation is a very useful tool to study the impact of small fields on ROF values. It can simulate the energy deposited in a material regardless of the field size. Although, detectors can be truly water equivalent and its dimensions can be adjusted to study detector size effects, but non-water equivalent detectors of small finite size would experience a partial volume effect in small fields because ionization is detected under non-charge particle equilibrium conditions and there will be a local disruption in the electron fluence.

This study focuses on ROF measurement of small megavoltage photon beams using different detectors (ionization and solid-state detectors) as well as film and calculation performed with the MC method. Both measurements and calculations were carried out at different SSDs and X-ray energies. It also investigated the water-detector volume effect with MC simulations.

\section{Materials and Methods}

An Elekta Synergy equipped with an Agility 160 MLC linear accelerator that can produce 6, 10, and $15 \mathrm{MV}$ beams, was used in this study. Small-field dosimetry was performed in two phases: in the first phase, ROFs were measured using different detectors. In the second phase, ROFs were calculated using the EGSnrc MC codes, BEAMnrc and DOSXYZnrc.

Five detectors were used in this study: (i) Ionization chamber (IBA CC01), (ii-iii) Solid state detectors (IBA EFD 3G Electron diode and PTW60019 micro-diamond), and (iv-v) films (Radiographic Kodak EDR2 and Gafchromic EBT2).

Table 1. The physical characteristics of the detectors used in this study.

\begin{tabular}{ccccccc}
\hline \hline Detector & $\begin{array}{c}\text { Density } \\
\mathbf{g c m}^{-3}\end{array}$ & $\begin{array}{c}\text { Volume } \\
\text { cc }\end{array}$ & $\begin{array}{c}\text { Cavity } \\
\text { material }\end{array}$ & Shape & $\begin{array}{c}\text { Thickness } \\
(\mathbf{m m})\end{array}$ & $\begin{array}{c}\text { Radius } \\
(\mathbf{m m})\end{array}$ \\
\hline $\mathrm{CC} 1$ & 0.0012 & 0.01 & Air & Cylindrical & 3.6 & 1.0 \\
$\mathrm{EFD}^{3 \mathrm{G}}$ & 2.3 & 0.0002 & $\begin{array}{c}\text { p-type } \\
\text { silicon }\end{array}$ & Circular & 0.06 & 2.0 \\
$\begin{array}{c}\text { PTW } \\
\text { diamond }\end{array}$ & 3.5 & 0.00004 & diamond & Circular & 0.001 & 1.1 \\
\hline \hline
\end{tabular}


Field sizes of $1 \times 1,2 \times 2,3 \times 3,4 \times 4,5 \times 5$ and (reference field) of $10 \times 10 \mathrm{~cm}^{2}$ were used for ROF measurements at 6,10 and $15 \mathrm{MV}$ for four SSDs 90, 95, 100 and $110 \mathrm{~cm}$. ROFs were normalized to the $10 \times 10 \mathrm{~cm}^{2}$ field at $100 \mathrm{~cm} \mathrm{SSD} \mathrm{for} \mathrm{each}$ beam energy under consideration. Measurements were carried out for 100 monitor units (MU) at $400 \mathrm{cGy} / \mathrm{min}$. Relative output factors (ROFs) were measured on the central axis $(\mathrm{CAX})$ at $10 \mathrm{~cm}$ depth in water for the IBA CC01, IBA EFD ${ }^{3 \mathrm{G}}$, and the PTW60019 microDiamond detectors. Solid water (RW3, Goettingen whitewater) was used for the EDR2 and EBT2 film measurements.

For the water-phantom measurements, the detectors were aligned on the CAX using Omnipro® Accept 6.4a (IBA Dosimetry GmbH, Schwarzenbruck, Germany) scanning software. The electrometer unit was warmed up and allowed to stabilize for 30 minutes before measurements.

The solid water phantom (RW3) has a water equivalent physical density of $1.045 \mathrm{~g} / \mathrm{cm}^{3}$. The elemental composition by relative weight is $7.59,90.41,0.8$ and $1.2 \%$ for Hydrogen, Carbon, Oxygen, and Titanium respectively. The radiographic EDR2 films which were used for measurement of ROFs were cut into $5 \mathrm{~cm} \times 20 \mathrm{~cm}$ film pieces and sealed within a lighttight envelope. The film response versus dose calibration curves (Pai et al., 2007) were measured for each photon beam energy using a $10 \times 10 \mathrm{~cm}^{2}$ field size, with several films placed at $10 \mathrm{~cm}$ depth in an RW3 phantom with a source to surface distance (SSD) of $100 \mathrm{~cm}$ and gantry angle of $0^{\circ}$. The dose given was $0,30,60,90,120$, and $150 \mathrm{cGy}$ at $400 \mathrm{cGy} / \mathrm{min}$ for all measurements [20].

The films were developed using a PROTEC OPTIMAX processor and scanned using a VIDAR VXR-12 plus scanner with a resolution of 75 dots per inch, at 12 bits depth, greyscale. Regions of interest were drawn at the centre of the film image using Image ${ }^{\circledR}$ software (a Java-based image analysis program U.S. National Institutes of Health) to obtain the average pixel value. The radiation dose given to the films was corrected using PDD values at $10 \mathrm{~cm}$ depth for 6,10 and $15 \mathrm{MV}$ taken as $67.5 \%, 72.0 \%$, and $76.5 \%$ respectively. The average pixel values along with the reconstructed doses at 10 $\mathrm{cm}$ depth were used to establish calibration curves [21]. The resulting dose and optical density (OD) were used to establish a fitting curve, which will allow the dose to be determined at any OD within the maximum range. The films that were used for measurement of ROFs were exposed to $120 \mathrm{MU}$ for different field sizes with the gantry at $0^{\circ}$.

The calibration procedure for the EBT2 was as follows: The calibration film pieces $\left(2 \times 2.5 \mathrm{~cm}^{2}\right)$ were exposed to 0,50 , 100, 200 and $250 \mathrm{cGy}$. These film pieces were placed in the centre of a $10 \times 10 \mathrm{~cm}^{2}$ field, with $10 \mathrm{~cm} \mathrm{RW3} \mathrm{build-up}$ $[22,23]$ and the gantry angle of $0^{\circ}$ at an SSD of $100 \mathrm{~cm}$. The 24-hour waiting period was observed after which the films were scanned using the Epson V330 document scanner. The scanning parameters that were used: 72 dots per inch (dpi), 48- bit colour depth [24] and all scanner enhancements were deactivated.

Scanned images were analysed using ImageJ. Regions of interest were drawn in at the centre of the exposed film pieces to obtain the average pixel value. The dose given was also corrected for depth as described above for the EDR2 radiographic film. The calibration curve was then determined for each colour [25]. The triple channel calibration method was used since it improves the accuracy of the measurement technique. It also corrects for variations in the thickness of the active layer, the scanner nonlinearity, and noise [26].

\section{Monte Carlo simulation}

An accurate model of the Elekta Synergy Linac head was modelled to simulate radiation transport through it with the BEAMnrc code [27,28]. Afterward, Phase Space data were used as input sources in the DOSXYZnrc MC code for particle transport and energy deposition in a water phantom. The photon and electron transport cut-offs (PCUT and ECUT) were $0.01 \mathrm{MeV}$ and $0.521 \mathrm{MeV}$, respectively. The maximum step size (SMAX) defines the maximum step electron in centimeter (cm). It was set to default since the restriction is not necessary when electron step algorithms PRESTA II and the EXACT boundary algorithm are in use. Also, the maximum fraction energy loss/step (ESTEPE) was set to 0.25 (25\%) for accurate electron transport. The number of histories was enough to reduce the variance to less than $1 \%$ in the useful beam area.

For the ROF study, an accurate source model was simulated for $1 \times 1,2 \times 2,3 \times 3,4 \times 4,5 \times 5$ and $10 \times 10 \mathrm{~cm}^{2}$ field at 6 , 10 and $15 \mathrm{MV}$ photon beams [29]. The field sizes were defined at $100 \mathrm{~cm} \mathrm{SSD.} \mathrm{ROFs} \mathrm{were} \mathrm{calculated} \mathrm{in} \mathrm{a} \mathrm{voxel} \mathrm{size} \mathrm{of}$ $0.2 \times 0.2 \times 0.2 \mathrm{~cm}^{3}$ defined in a water tank $\left(40 \times 40 \times 40 \mathrm{~cm}^{3}\right)$ for 90, 95, 100 and $110 \mathrm{~cm} \mathrm{SSD.} \mathrm{The} \mathrm{simulated} \mathrm{ROFs} \mathrm{were}$ benchmarked with measured data obtained in this study. The further study evaluated the effect of voxel sizes on the ROFs by changing the voxel sizes to $0.1 \times 0.1 \times 0.1$ and $0.5 \times 0.5 \times 0.5 \mathrm{~cm}^{3}$ at $100 \mathrm{SSD}$.

All simulations in this study were carried out on a Linux OS workstation super microcomputer, equipped with 24 Intel(R) Xeon(R) core processors, each with CPU time of $2.10 \mathrm{GHz}$ and 64 GB RAM.

\section{Results}

In Figures 1-3 the ROF increases with an increase in field size. The MC obtained ROF values are within the range of the ROF values measured with the five different detectors. The most substantial ROF variation occurs at the $1 \times 1 \mathrm{~cm}^{2}$ field size. This field size is where the detectors used in the water measurements experienced the largest variations even with realignment check (their signals depended strongly on the alignment with the central beam axis of the detectors). 
(a) ROF at $90 \mathrm{~cm} \mathrm{SSD} \mathrm{for} 6 \mathrm{MV}$

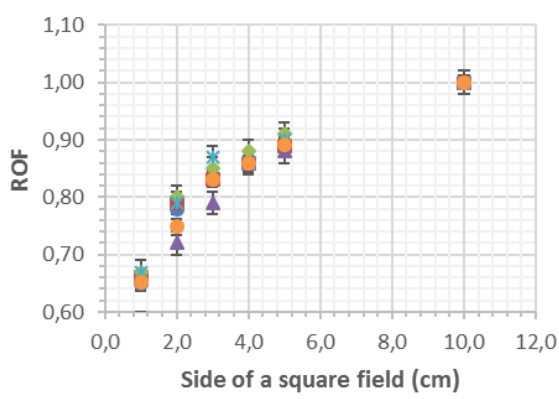

- $\mathrm{CC01}$ EFD-3G $\diamond$ PTW60019 $\Delta$ EBT2 $*$ EDR2 $\bullet \mathrm{MC}$

(b) ROF at $95 \mathrm{~cm}$ SSD for $6 \mathrm{MV}$

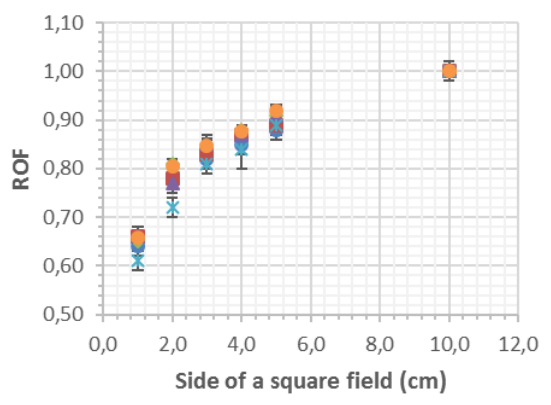

- $\mathrm{CC01}$ EFD-3G $\diamond \mathrm{PTW60019} \Delta \mathrm{EBT} 2 * \mathrm{EDR} 2 \bullet \mathrm{MC}$

(c) ROF at $100 \mathrm{~cm} \mathrm{SSD} \mathrm{for} 6 \mathrm{MV}$

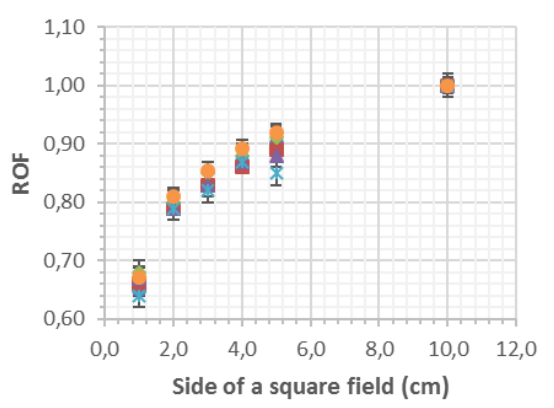

- CC01 —EFD-3G PTW60019 $\Delta$ EBT2 *EDR2 - MC

(d) ROF at $110 \mathrm{~cm} \mathrm{SSD} \mathrm{for} 6 \mathrm{MV}$

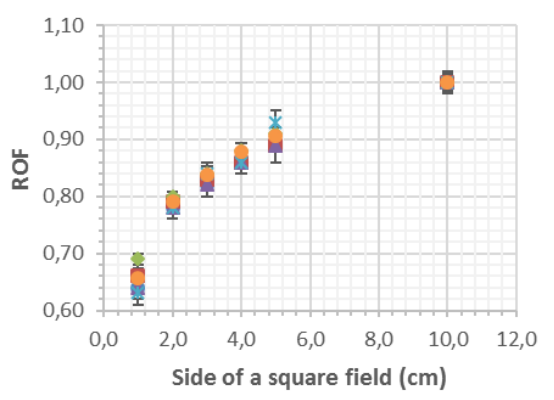

- CC01 —EFD-3G • PTW60019 $\Delta$ EBT2 *EDR2 - MC

Figure 1. Relative output factors measured for the different detectors and MC for a 6 MV photon energy beam. The SSD was set at (a) $90 \mathrm{~cm}$, (b) $95 \mathrm{~cm}$, (c) $100 \mathrm{~cm}$ and, (d) $110 \mathrm{~cm}$. ROF values were normalized to the individual detector values for the $10 \times 10$ $\mathrm{cm}^{2}$ field. (a) ROF at $90 \mathrm{~cm} \mathrm{SSD} \mathrm{for} 10 \mathrm{MV}$

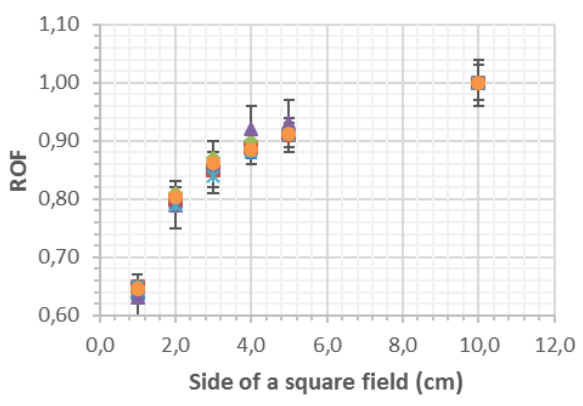

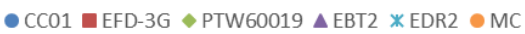

(b) ROF at $95 \mathrm{~cm} \mathrm{SSD} \mathrm{for} 10 \mathrm{MV}$

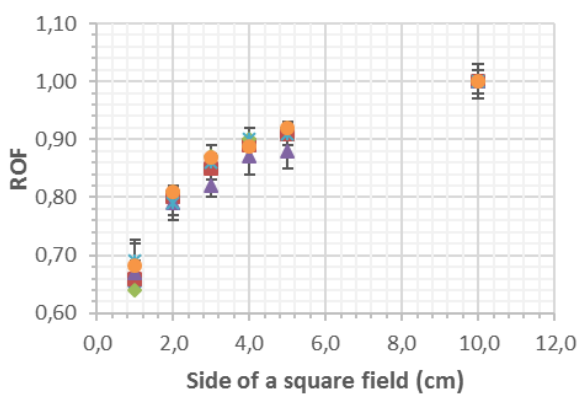

- CC01 EFD-3G P PTW60019 $\Delta$ EBT2 $*$ EDR2 - MC

(c) ROF at $100 \mathrm{~cm} \mathrm{SSD} \mathrm{for} 10 \mathrm{MV}$

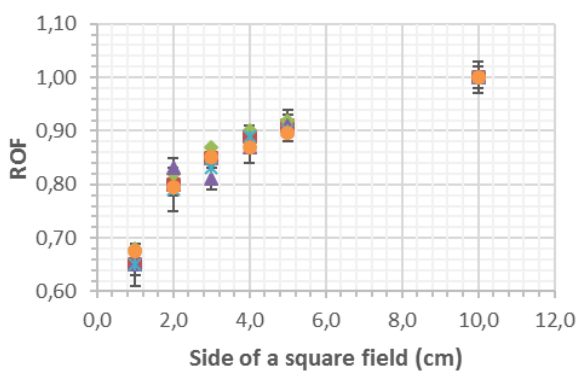

- CC01 —EFD-3G • PTW60019 $\Delta$ EBT2 $*$ EDR2 - MC

(d) ROF at $110 \mathrm{~cm} \mathrm{SSD} \mathrm{for} 10 \mathrm{MV}$

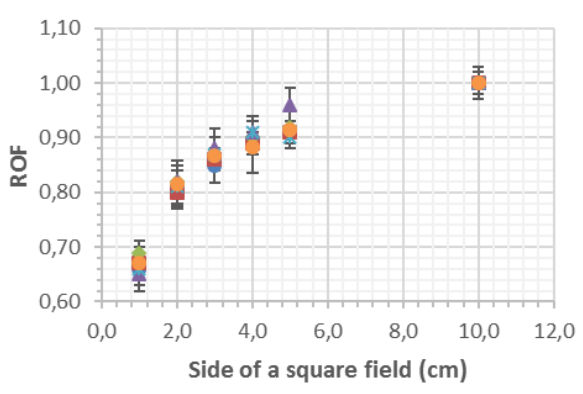

- CC01 —EFD-3G PTW60019 \ EBT2 *EDR2 — MC

Figure 2. Relative output factors measured for the different detectors and MC for a 10 MV photon energy beam. The SSD was set at (a) $90 \mathrm{~cm}$, (b) $95 \mathrm{~cm}$, (c) $100 \mathrm{~cm}$ and, (d) $110 \mathrm{~cm}$. ROF values were normalized to the individual detector values for the $10 \times 10$ $\mathrm{cm}^{2}$ field. (a) ROF at $90 \mathrm{~cm} \mathrm{SSD} \mathrm{for} 15 \mathrm{MV}$

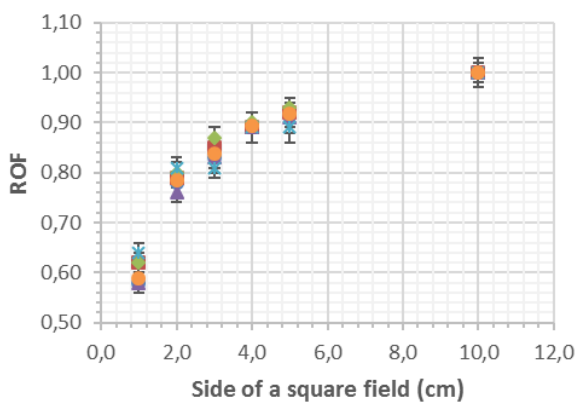

- CC01 EFD-3G PTW60019 А EBT2 *EDR2 - MC

(b) ROF at $95 \mathrm{~cm} \mathrm{SSD} \mathrm{for} 15 \mathrm{MV}$

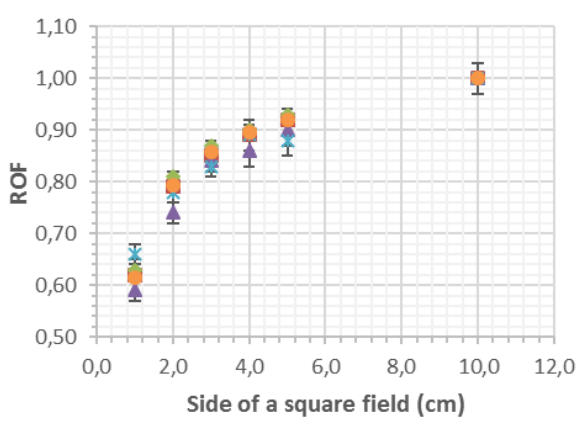

- $\mathrm{CC01}$ EFD-3G $\bullet$ PTW60019 $\Delta$ EBT2 $*$ EDR2 ・ MC

(c) ROF at $100 \mathrm{~cm} \mathrm{SSD} \mathrm{for} 15 \mathrm{MV}$

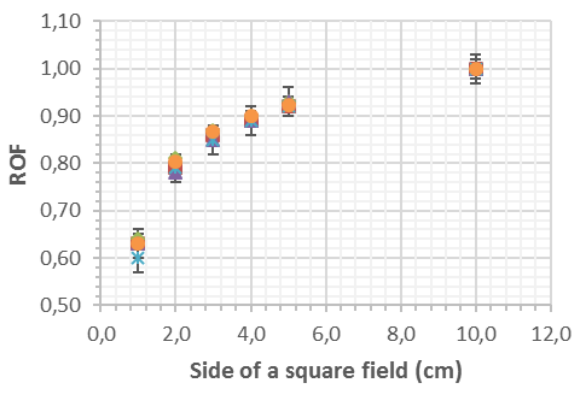

- CC01 —EFD-3G PTW60019 \ EBT2 *EDR2 - MC

(d) ROF at $110 \mathrm{~cm} \mathrm{SSD} \mathrm{for} 15 \mathrm{MV}$

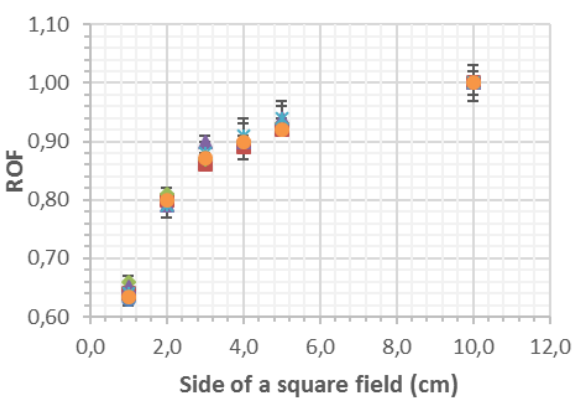

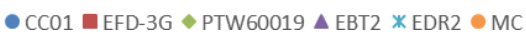

Figure 3. Relative output factors measured for the different detectors and MC for a $\mathbf{1 5}$ MV photon energy beam. The SSD was set at (a) $90 \mathrm{~cm}$, (b) $95 \mathrm{~cm}$, (c) $100 \mathrm{~cm}$ and, (d) $110 \mathrm{~cm}$. ROF values were normalized to the individual detector values for the $10 \times 10$ $\mathrm{cm}^{2}$ field. 
A previous study indicated that the minimum square field size to obtain lateral electronic equilibrium for 6,10 and $15 \mathrm{MV}$ beams is $2.6 \times 2.6 \mathrm{~cm}^{2}, 3.4 \times 3.4 \mathrm{~cm}^{2}$, and $3.8 \times 3.8 \mathrm{~cm}^{2}(\mathrm{Li}$ et al. 1995). Lateral electronic disequilibrium effect can be observed for fields smaller than $4 \times 4 \mathrm{~cm}^{2}$ in Figures 1-3. The signal measured at a point is due to the influence of the primary beam and scatter radiation. As the field size gradually decreases so does the influence of scattering at the central axis, or the point of measurement, resulting in a decrease of the signal measured thus a decrease in the ROF. Once the lateral electronic equilibrium minimum field size is decreased, the decline in the measured signal is steeper resulting in a sharper drop of the ROF below the $4 \times 4 \mathrm{~cm}^{2}$ field.

In Figure 4, the change in ROF per unit square field size (ROF value was divided by the side of its square field) is plotted at $100 \mathrm{~cm} \mathrm{SSD}$ for 6,10 and $15 \mathrm{MV}$ photon beams. It shows the rate of change in the ROF concerning field size is at its highest when going from the $1 \times 1 \mathrm{~cm}^{2}$ to the $2 \times 2 \mathrm{~cm}^{2}$ field (first data point set on each graph). This indicates the largest disruption in lateral electron equilibrium. There are small variations among the detectors used in the ROF measurements. The energy effect is more pronounced as the average differential ROF varies from 0.045 at $6 \mathrm{MV}$ to 0.049 at $10 \mathrm{MV}$ and 0.055 at $15 \mathrm{MV}$ at the $1 \times 1 \mathrm{~cm}^{2}$ field. The disruption of later electronic equilibrium is more pronounced at $15 \mathrm{MV}$. At fields larger than $5 \times 5 \mathrm{~cm}^{2}$ the differential ROF gets much smaller due to the attainment of later electronic equilibrium and complete scattering from the treatment head of the linac.

Figure 5 shows the ROF variation (at $100 \mathrm{~cm} \mathrm{SSD)}$ as a function of detector density, here the film data were excluded, and only MC and detector data were used. It is evident and known that a field size effect is present (as shown in Figures 14). In Figure 5 there is also an indication that the PTW10016 diamond detector was the most sensitive in all three graphs.

A fitting function of the form:

$R O F=a S^{b} \operatorname{Tanh}(c S)$

Eq. 1

Was used to parameterize the ROF where $a, b$ and $c$ are fitting constants, and $\mathrm{S}$ is the square field side length. The ROF data was fitted with a deviation within $1.5 \%$ for most cases as shown in Figure 6 for the CC01 chamber as an example. The fitting constants are shown in Table 1. These constants also depend on the detector used.

Table 1. Fitting constants used in Equation 1 for the indicated beam energies.

\begin{tabular}{cccc}
\hline \hline Energy (MV) & $\mathbf{a}\left(\mathbf{c m}^{-\mathbf{1}}\right)$ & $\mathbf{b}$ & $\mathbf{c}\left(\mathbf{c m}^{-\mathbf{1}}\right)$ \\
\hline 6 & 0.730 & 0.135 & 1.500 \\
10 & 0.765 & 0.120 & 1.250 \\
15 & 0.780 & 0.110 & 1.200 \\
\hline \hline
\end{tabular}

(a) ROF/SF for $6 \mathrm{MV}$

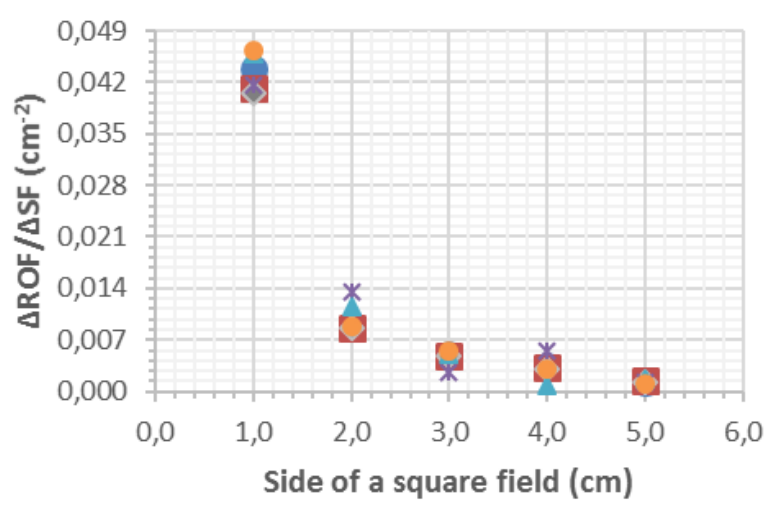

- $\mathrm{CCO}$ —EFD-3G \& PTW60019 * EBT2 $\triangle \mathrm{EDR} 2 \bullet \mathrm{MC}$

(b) ROF/SF for $10 \mathrm{MV}$

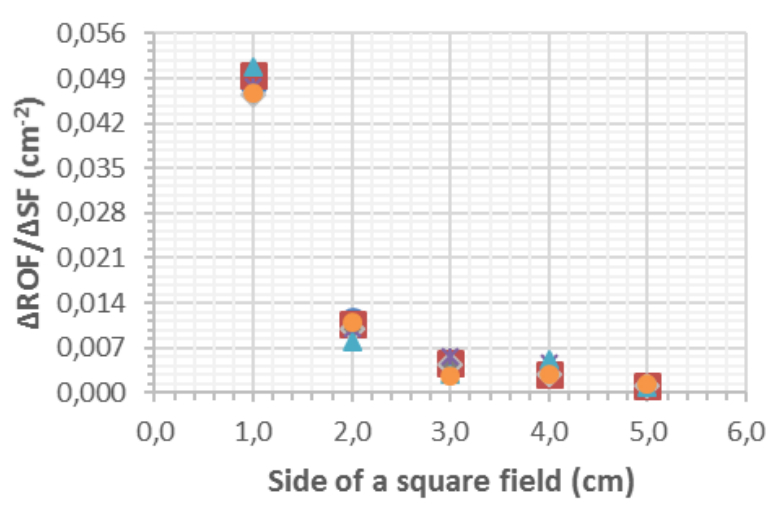

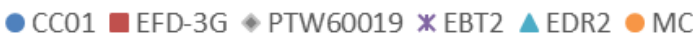

(c) ROF/SF for $15 \mathrm{MV}$

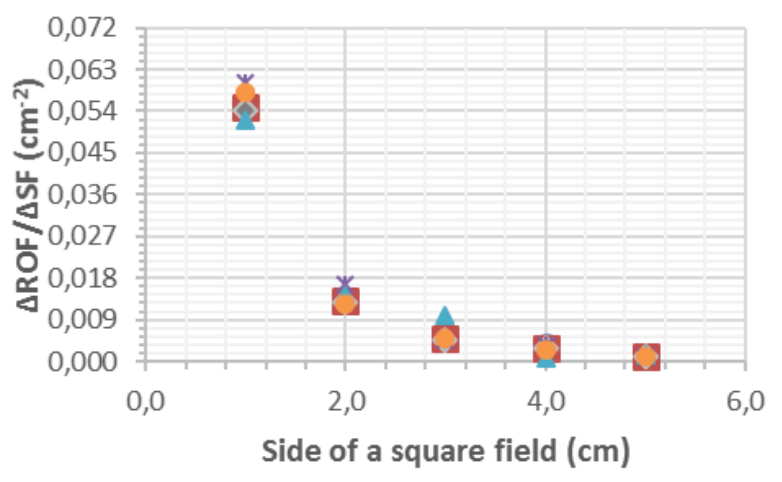

- $\mathrm{CC01}$ EFD-3G + PTW60019 * EBT2 $\triangle \mathrm{EDR} 2$ - MC

Figure 4. The change in differential ROF for each detector at 100 cm SSD (a) for $6 \mathrm{MV}$, (b) for $10 \mathrm{MV}$, and (c) for $15 \mathrm{MV}$. 
(a) ROF vs Density for 6 MV

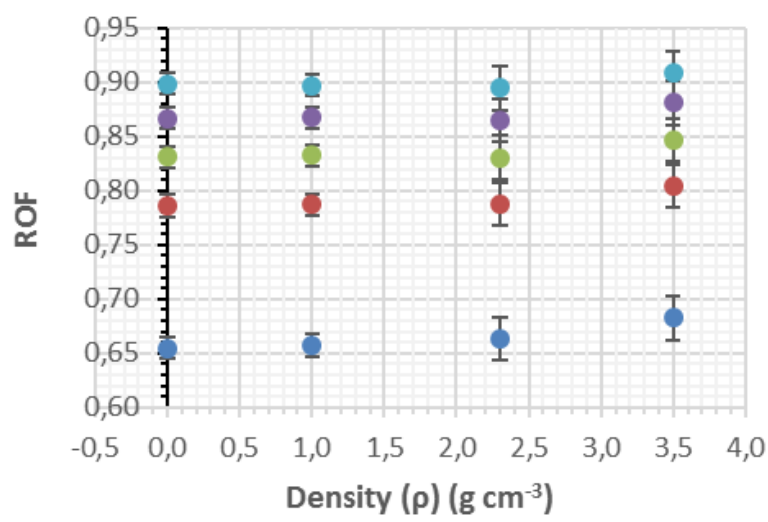

- $1 \times 1 \mathrm{~cm} \mathrm{SF} \bullet 2 \times 2 \mathrm{~cm} \mathrm{SF} \bullet 3 \times 3 \mathrm{~cm} \mathrm{SF}$

- $4 \times 4 \mathrm{~cm} \mathrm{SF} \bullet 5 \times 5 \mathrm{~cm} \mathrm{SF}$

(b) ROF vs Density for $10 \mathrm{MV}$

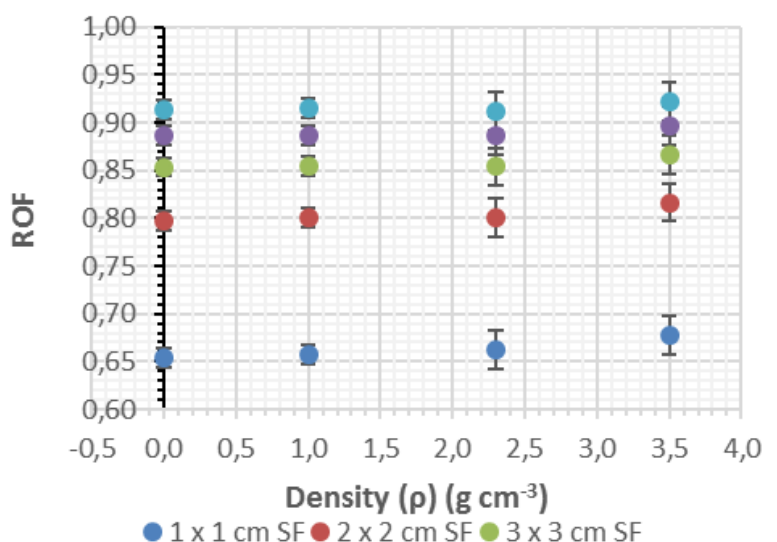

$\bullet 4 \times \mathrm{cm} \mathrm{SF} \bullet 5 \times 5 \mathrm{~cm} \mathrm{SF}$

(c) ROF vs Density for 15 MV

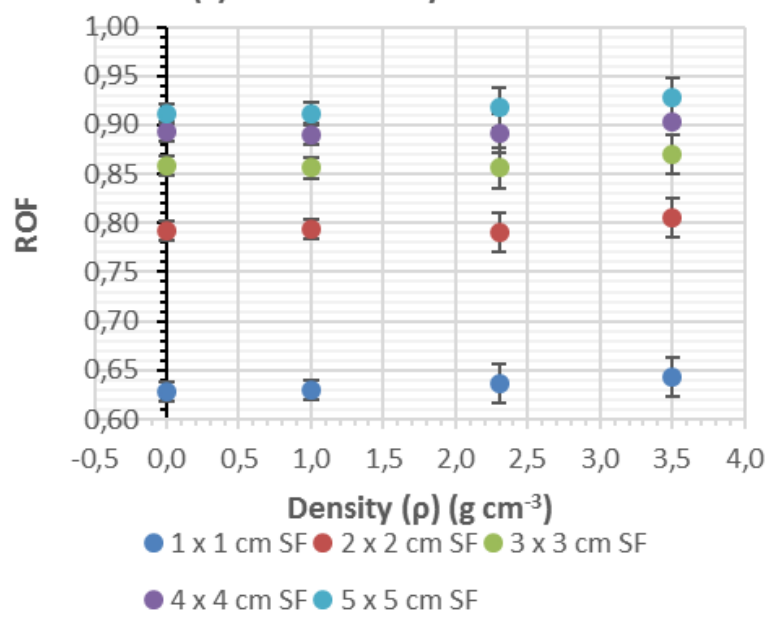

Figure 5. Density effect of $\mathrm{CCO1}\left(0.0012 \mathrm{gcm}^{-3}\right)$, MC water phantom $\left(1 \mathrm{gcm}^{-3}\right)$, EFD-3G $\left(2.3 \mathrm{gcm}^{-3}\right)$, and PTW10016 $\left(3.5 \mathrm{gcm}^{-}\right.$ ${ }^{3}$ ) detectors on ROF data for (a) $6 \mathrm{MV}$, (b) $10 \mathrm{MV}$, and (c) $15 \mathrm{MV}$ at $100 \mathrm{~cm}$ SSD. (a) ROF at $100 \mathrm{~cm} \mathrm{SSD} \mathrm{for} 6 \mathrm{MV}$

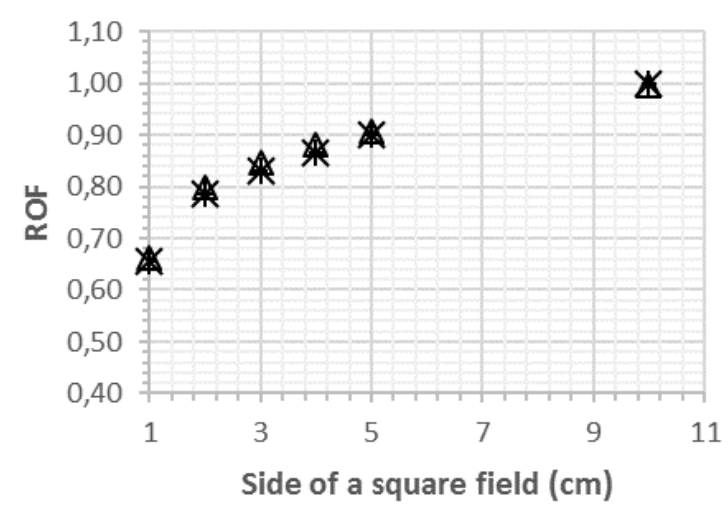

* CCO1 $\Delta$ BFit

(b) ROF at $100 \mathrm{~cm} \mathrm{SSD} \mathrm{for} 10 \mathrm{MV}$

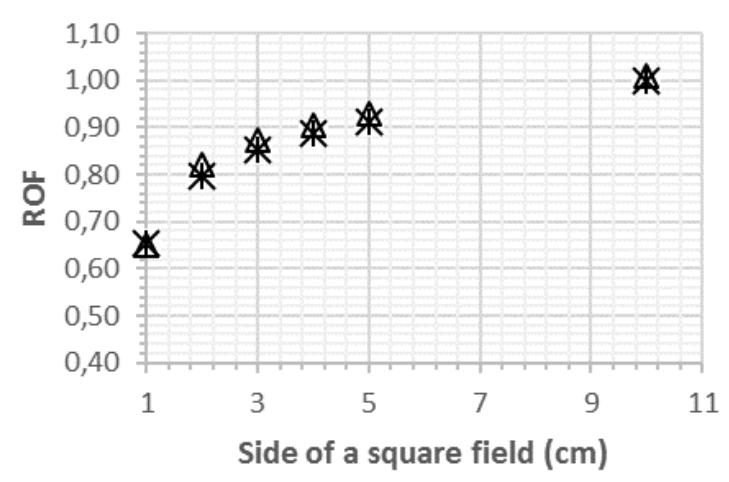

$\boldsymbol{*}$ CCO1 $\Delta$ BFit

(c) ROF at $100 \mathrm{~cm} \mathrm{SSD} \mathrm{for} 15 \mathrm{MV}$

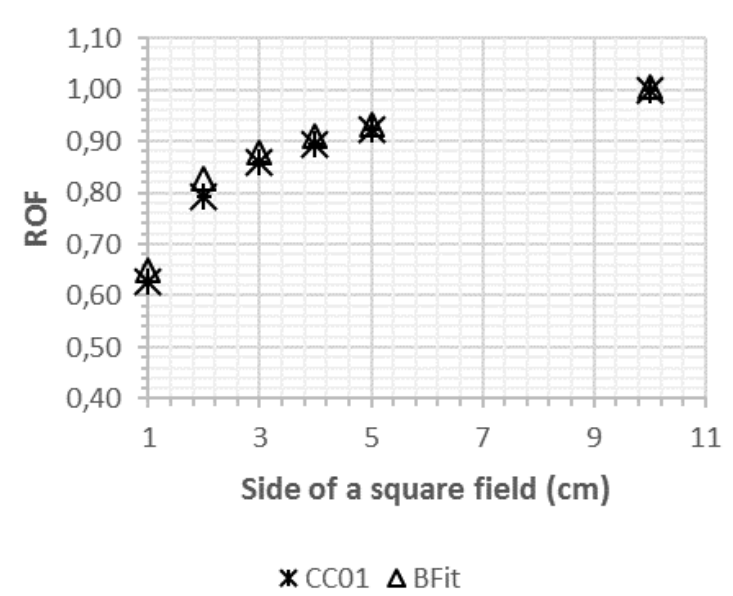

Figure 6. Evaluation of fitting ROF for (a) 6, (b) 10 and, (c) 15 MV photon beams for ROF data for measurements for the CC01 detector and MC simulations at 100 SSD. 


\section{Discussion}

The ROF data were measured for small fields at different photon energies and different detectors, film, and EGSnrc MC simulations at $90 \mathrm{~cm}, 95 \mathrm{~cm}, 100 \mathrm{~cm}$ and $110 \mathrm{~cm}$ SSDs. For the water phantom setup, it was found that accurate center of the CC01, EFD-3G, and PTW10016 detectors was important since the beam profile is very narrow at smaller fields. Few articles have used convolution and deconvolution kernel to measure finite detector response to photon beams. Using Gaussian onedimensional kernels, Fourier transforms, and Hermite polynomial expansions, the articles concluded that detector's influence plays a major role in relative and absolute dose calculation [30-32].

It was necessary to re-centre the detector for the smallest field sizes of $1 \times 1 \mathrm{~cm}^{2}$ as the focal spot can move around when changing from one energy to the next $[7,33]$.

From the observations in Figures 1-3, the spread in the detector and film ROF data is in the order of 8 percent. ROFs are shown to be dependent on the incoming photon beam energy, field size, and density of the detectors studied. The rate of ROF change per unit field size is the largest for the smallest field studied. The rate of ROF change per unit density is the largest for the most-dense detector (PTW10016). This was observed across all fields studied and the effect enhanced at $1 \times 1 \mathrm{~cm}^{2}$ and $2 \times 2 \mathrm{~cm}^{2}$ fields. Here lateral electronic equilibrium is perturbed by the small field, and the density effect of the PTW10016 chamber also enhanced local electron equilibrium disruption. The PTW10016 detector tended to yield higher ROF values as shown in figure 5 if the MC data are taken as the benchmark for the unit density water detector. It is interesting to see that the air chamber, $\mathrm{CC} 01$, is the closest water equivalent model among those used in this study. Film data were not included in the analysis in Figures 5 and 6.

Previously, Scott et al. [33] varied the density effect on ROF data for diamond, silicon and air density at a depth of $5 \mathrm{~cm}$ using MC simulation. The study stated that variations in detector signal response to small field dosimetry depends on densities of detector active volume and slightly independent of the atomic number of the detector's material. Similarly, Underwood et al. [10] stated that the detector's response is principally influenced by its density and that the density around the detector's active volume contributes to the detector's signal response.

The ROF versus field size could be fitted to a very high degree of accuracy with Equation 1. What makes it very attractive is its ability to fit ROF data in this study from the $1 \times 1 \mathrm{~cm}^{2}$ to the $10 \times 10 \mathrm{~cm}^{2}$ with a single continuous function as shown in Figure 7. (a) ROF at $100 \mathrm{~cm} \mathrm{SSD} \mathrm{for} 6 \mathrm{MV}$

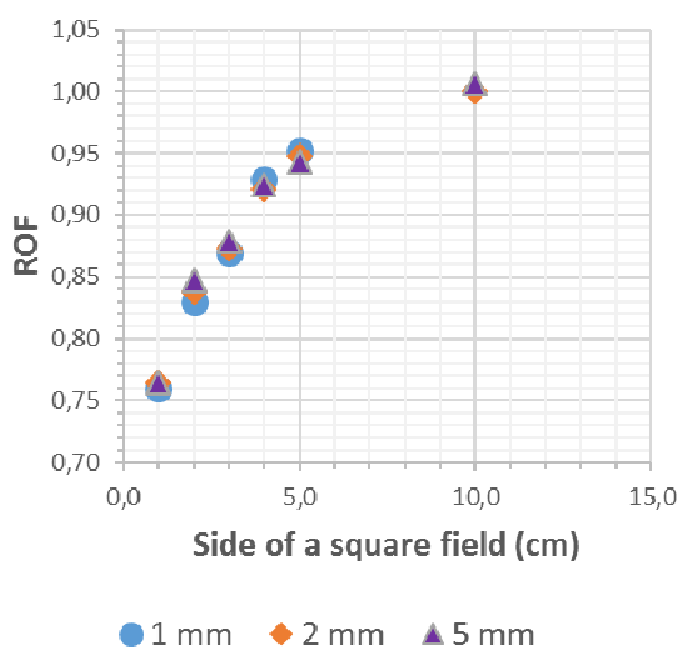

(b) ROF at $100 \mathrm{~cm} \mathrm{SSD} \mathrm{for} 10 \mathrm{MV}$

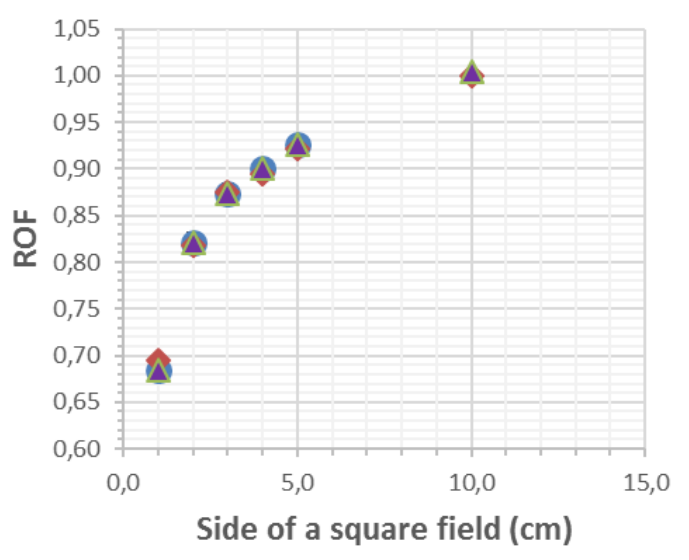

$1 \mathrm{~mm} \diamond 2 \mathrm{~mm} \Delta 5 \mathrm{~mm}$

(c) ROF at $100 \mathrm{~cm}$ SSD for $15 \mathrm{MV}$

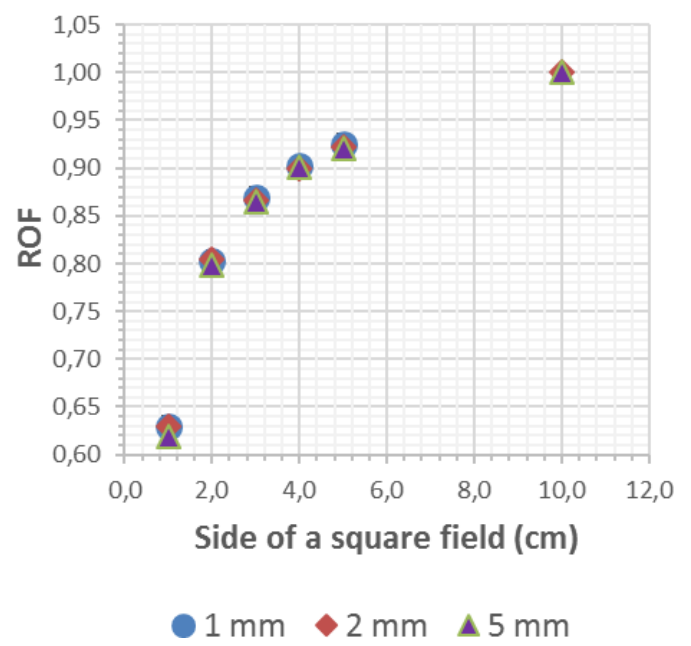

Figure 7. ROF simulated data for 1, 2 and $5 \mathrm{~mm}$ side of a voxel at $100 \mathrm{SSD}$ of a voxel at (a) $6 \mathrm{MV}$, (b) $10 \mathrm{MV}$, and (c) $15 \mathrm{MV}$ photon beams. 


\section{(a) ROF vs Energy for $1 \mathrm{~mm}$ side of a voxel}

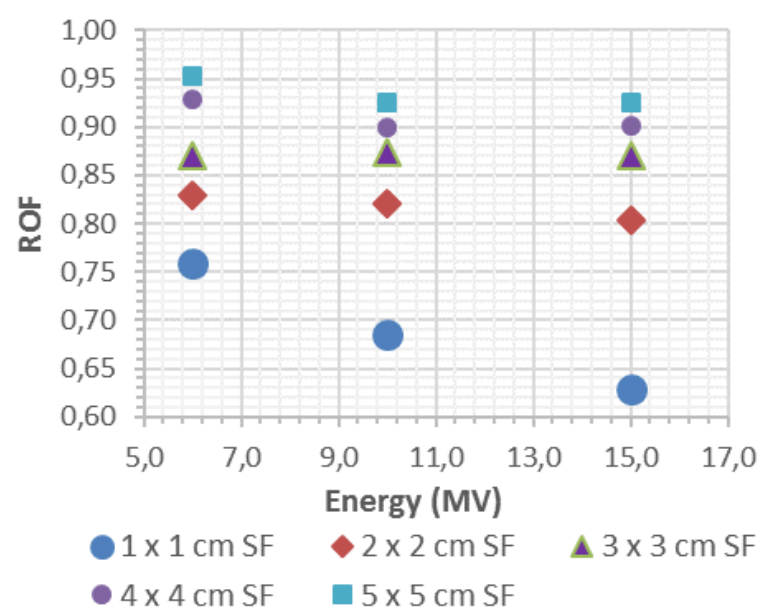

(b) ROF vs Energy for $5 \mathrm{~mm}$ side of a voxel

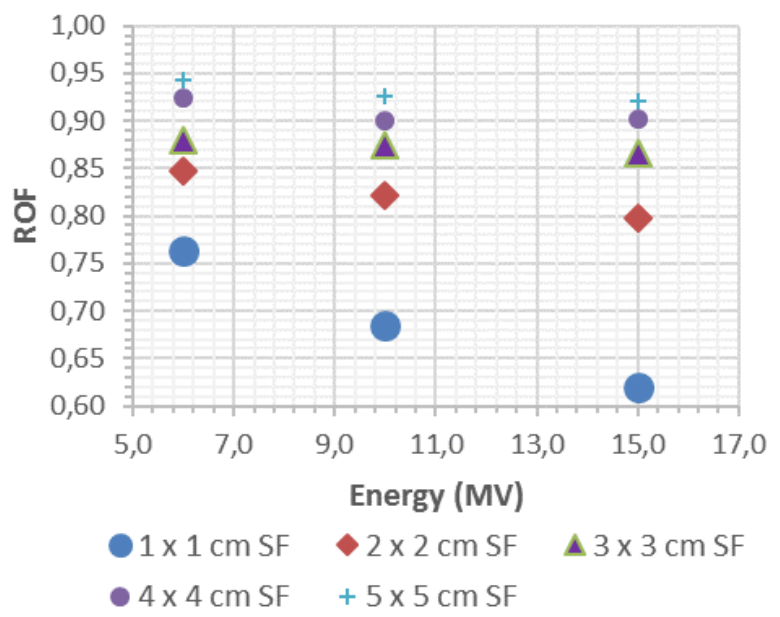

Figure 8. ROF simulated data for 6,10 and $15 \mathrm{MV}$ photon beams at $100 \mathrm{SSD}$ for (a) $1 \mathrm{~mm}$ and (b) 5 mm side of a voxel.

\section{Monte Carlo simulation and the effect of voxel size}

$\mathrm{ROF}$ data for the MC simulations were evaluated from a voxel size of $2 \times 2 \times 2 \mathrm{~mm}^{3}$ that is used conventionally. Further study on this evaluated the effect of changing the voxel sizes. The ROF data were normalized at $10 \times 10 \mathrm{~cm}^{2}$ field for a $2 \mathrm{~mm}$ voxel side length for 6, 10 and $15 \mathrm{MV}$. In Figure 7, the increase in voxel sizes did not indicate that the ROF values will differ significantly at any given field size. It is interesting that calculations carried out even for the largest voxel $(5 \times 5 \times 5$ $\mathrm{mm}^{3}$ ) gave the same ROF. The water detector does not disrupt local electronic equilibrium, and its size does not seem to be influenced by the field dimensions as seen in Figure 8 for the smallest field at varying water detector dimensions. Laub and Wong [34] work has previously stated that the water-equivalent detector is essential for output factor measurement of small fields. Similarly, another study has shown a slightly significant response to changing the voxel sizes for water detector. However, silicon detector shows a significant response to changing voxel sizes since its non-water density material whereby the perturbation effect is pronounced [33]. It thus seems that the determining factor in detector response at smaller fields is their physical composition and density that disrupt local electron fluence, compared to water. In Figure 8, the loss due to electron scattering becomes conspicuous at high energy, especially for $1 \times 1 \mathrm{~cm}^{2}$ field. With more electron moving out of the field, there will be less dose deposited within the active detector volume [6]. Therefore, there will be a sharp fall of ROF values at smaller field sizes and high-energy beams.

Figure 8 shows how the MC calculated ROF values are influenced by beam energy for the stated square field sizes. It is noted that the ROF shows the greatest sensitivity for the $1 \times 1$ $\mathrm{cm}^{2}$ field size, the size of water detector did not influence it when inspecting the data sets in Figure 8a to corresponding data in Figure 8b. Thus for a pure water detector, the energy dependency is strongest for smaller fields. The total change in ROF is 0.16 units between 6 and $15 \mathrm{MV}$ for the $1 \times 1 \mathrm{~cm}^{2}$ field in figure 8. ROF data at $100 \mathrm{~cm} \mathrm{SSD}$ in Figures 1-3 show an average decline in ROF data of 0.08 units overall detectors used in this study.

\section{Conclusions}

The sensitive factors that influenced the ROF were the field size where the ROF rate of change per unit field length is highest at $1 \times 1 \mathrm{~cm}^{2}$ (smallest) field. Apart from films, EFD and diamond detectors have the highest density effect. For an ideal water detector (MC simulation), the ROF was only dependent on beam energy and field size, but not on the range of detector sizes $\left(1 \mathrm{~mm}^{3}-125 \mathrm{~mm}^{3}\right)$. The $1 \times 1 \mathrm{~cm}^{2}$ field showed the largest variation in ROF over the beam energies studied (Figure 8). Small high-density detectors may have a good sampling signal in a small beam, but their density effect causes larger ROF as seen in Figure 5 for the $1 \mathrm{~g} / \mathrm{cm}^{3}$ water detector data included.

\section{Acknowledgement}

This research project was funded by the South Africa Medical Research Council (SAMRC) with funds from the National Treasury under its Economic Competitive and Support package. This research and the publication thereof is the result of funding provided by the SAMRC under the High Energy Advanced Radiation (HARD) sponsorship programme SAMRC-RFA-UFSP-01-2013/HARD. 


\section{References}

[1] Benedict SH, Yenice KM, Followill D, et al. Stereotactic body radiation therapy: The report of AAPM Task Group 101. Med Phys. 2010;37(8):4078-4101.

[2] Godwin GA, Mugabe K. Characterization of a dynamic multi-leaf collimator for stereotactic radiotherapy applications. Phys Med Biol. 2012;57(14):4643-4654.

[3] Russo S, Reggiori G, Cagni E, et al. Small field output factors evaluation with a microDiamond detector over 30 Italian centers. Phys Med. 2016;32(12):1644-1650.

[4] Das IJ, Ding GX, Ahnesjo A. Small fields: Nonequilibrium radiation dosimetry. Med Phys. 2008;35(1): 206-215.

[5] Cranmer-Sargison G, Weston S, Sidhu NP, Thwaites DI. Experimental small field 6 MV output ratio analysis for various diode detector and accelerator combinations. Radiother Oncol. 2011;100(3):429-435.

[6] Gagnon JC, Theriault D, Guillot M, et al. Dosimetric performance and array assessment of plastic scintillation detectors for stereotactic radiosurgery quality assurance. Med Phys. 2012;57(14):429-436.

[7] Scott AJ, Nahum AE, Fenwick JD. Monte Carlo modeling of small photon fields: Quantifying the impact of focal spot size on source occlusion and output factors, and exploring miniphantom design for small-field measurements. Med Phys. 2009;26(7):3132-3144.

[8] Herrup D, Chu J, Cheung H, Pankuch M. Determination of penumbral widths from ion chamber measurements. Med Phys. 2005;32(12):3636-3640.

[9] Nasir MKR, Amjad N, Razzaq A, Siddique MT. Measurement and Analysis of PDDs Profile and Output Factors for Small Field Sizes by cc13 and Micro-Chamber cc01. International Journal of Medical Physics, Clinical Engineering and Radiation Oncology. 2017;6(1):36-56.

[10] Underwood TS, Winter HC, Hill MA, Fenwick JD. Detector density and small field dosimetry: Integral versus point dose measurement schemes. Med Phys. 2013;40(8):1-16.

[11] Sauer OA, Wilbert J. Measurement of output factors for small photon beams. Med Phys. 2007;34(6): 1983-1988.

[12] Fox C, Simon T, Simon B, et al. Assessment of the setup dependence of detector response functions for mega-voltage linear accelerators. Med Phys. 2010;37(2):477-484.

[13] Low D, Morgan J, Dempsey J, et al. Dosimetry tools and techniques for IMRT. Med Phys. 2011;38(3):1313-1338.

[14] Tyler MK, Liu PZ, Lee C, et al. Small field detector correction factors: effects of the flattening filter for Elekta and Varian linear accelerators. J Appl Clin Med Phys. 2016;17(3):223-235.

[15] Pai S, Das IJ, Dempsey JF, et al. TG-69: Radiographic film for megavoltage beam dosimetry. Med Phys. 2007;34(6):2228-2258.

[16] Andres C, Castilo A, Tortosa R, et al. A comprehensive study of the Gafchromic EBT2 radiochromic film. A comparison with EBT. Med Phys. 2010;37(12):6271-6278.

[17] Mayles P, Nahum A, Rosenwald J. Handbook of Radiotherapy Physics: Theory and Practice. Taylor and Francis group, 2007.

[18] ISP. Gafchromic EBT2 Self-developing film for radiotherapy dosimetry. 1361 Alps Road Wayne, New Jersey, USA, 2009.

[19] Aland T, Kairn T, Kenny J. Evaluation of a Gafchromic EBT2 film dosimetry system for radiotherapy quality assurance. Australas Phys Eng Sci Med. 2011;34(2):251-260.

[20] Chetty IJ, Charland PM. Investigation of Kodak Extended Dose Range (EDR) Film for Megavoltage Photon Beam Dosimetry. Phys Med Biol. 2002;47(20):3629-3641.

[21] Shi C, Papanikolaou N, Yan Y, et al. Analysis of the Sources of Uncertainty for EDR2 Film-Based IMRT Quality Assurance. J App Clin Med Phys. 2006;37(2):1-8.

[22] Lewis D, Micke A, Yu X, Chan MF. An Efficient Protocol for Radiochromic Film Dosimetry Combining Calibration and Measurement in a Single Scan. Med Phys. 2012;39(10):6339-6350.

[23] Alfonso R, Andreo P, Capote R, et al. A New Formalism for Reference Dosimetry of Small and Nonstandard Fields. Med Phys. 2008;35(11):5179-5186.

[24] Hu Y, Wang Y, Fogarty G, Liu G. Developing a Novel Method to Analyse Gafchromic EBT2 Films in Intensity Modulated Radiation Therapy Quality Assurance. Australas Phys Eng Sci Med. 2013;36(4):487-494.

[25] Mendez I, Peterlin R, Hudej R, et al. On Multichannel Film Dosimetry with Channel-Independent Perturbations. Med Phys. 2014;41(1):11705.

[26] Micke A, Lewis DF, Yu X. Multichannel Film Dosimetry with Nonuniformity Correction. Med Phys. 2011;38(5):2523-2534.

[27] Kawrakow I, Mainegra-Hing E, Rogers DWO, et al. The EGSnrc code system: Monte Carlo simulation of electron and photon transport," NRCC PIRS-701, 2013.

[28] Rogers DWO. Fifty years of Monte Carlo simulations for medical physics. Phys Med Biol. 20061;51(13):R287-R301.

[29] Oderinde OM, du Plessis FCP. Technical note: A new wedge-shaped ionization chamber component module for BEAMnrc to model the integral quality monitoring system®. Radiat Phys Chem. 2017;141:346-351. 
[30] Ulmer W, Kaissl W. The inverse problem of a Gaussian convolution and its application to the finite size of the measurement chambers/detectors in photon and proton dosimetry. Phys Med Biol. 2003;48(6):707-727.

[31] García-Vicente F, Delgado JM, Rodríguez C. Exact analytical solution of the convolution integral equation for a general profile fitting function and Gaussian detector kernel. Phys Med Biol. 2000;45(3):645-650.

[32] García-Vicente F, Delgado JM, Peraza C. Experimental determination of the convolution kernel for the study of the spatial response of a detector. Med Phys. 1998;25(2):202-207.

[33] Scott AJ, Kumar S, Nahum AE, Fenwick JD. Characterizing the influence of detector density on dosimeter response in nonequilibrium small photon fields. Phys Med Biol. 2012;57(14):4461-4476.

[34] Laub WU, Wong T. The volume effect of detectors in the dosimetry of small fields used in IMRT. Med Phys. 2003;30(3):341-347. 